\title{
Retraction Note: Prediction of economic growth by extreme learning approach based on science and technology transfer
}

\author{
Petra Karanikić ${ }^{1}$ Igor Mladenović ${ }^{2}$ S Svetlana Sokolov-Mladenović ${ }^{2}$. \\ Meysam Alizamir ${ }^{3}$
}

Published online: 7 February 2019

(c) Springer Nature B.V. 2019

\section{Retraction Note: Qual Quant (2017) 51:1395-1401 https://doi.org/10.1007/s11135-016-0337-y}

The Editor-in-Chief has retracted this article (Karanikić et al. 2016) because validity of the content of this article cannot be verified. This article showed evidence of substantial text overlap [most notably with the articles cited (Marković et al. 2016; Mladenović et al. 2016a, b)], peer review and authorship manipulation. All of the authors disagreed with the publication of this retraction.

\section{References}

Karanikić, P., Mladenović, I., Sokolov-Mladenović, S., Alizamir, M.: Prediction of economic growth by extreme learning approach based on science and technology transfer (2016). Published 29 March 2016. https://urldefense.proofpoint.com/v2/url?u=https-3A_doi.org_10.1007_s11135-2D016-2D033 7-2Dy\&d=DwIFaQ\&c=vh6FgFnduejNhPPD0fl_yRaSfZy8CWbWnIf4XJhSqx8\&r=mImVkBpa54 MY3KIaquiNBAiNRIkbDDT3A207pVhZWFk\&m=61Q4FOkv2cm-bbmO9NhTz4lpNXaI3Xj1FqJBGzzgVc\&s=O1WO3uQCLvLZqs-NwgnKahkt8JZXTT1ZK7uPAwFuDcQ\&e=

Marković, D., Mladenović, I., Milovančević, M.: Estimation of the most influential science and technology factors for economic growth forecasting by soft computing technique (2016). Published 20 February 2016. https://urldefense.proofpoint.com/v2/url?u=https-3A_doi.org_10.1007_s11135-2D016-2D032 $1-2 D 6 \& d=D w I F a Q \& c=v h 6 F g F n d u e j N h P P D 0 f l \_y R a S f Z y 8 C W b W n I f 4 X J h S q x 8 \& r=m I m V k B p a 54$ MY3KIaquiNBAiNRIkbDDT3A207pVhZWFk\&m=61Q4FOkv2cm-bbmO9NhTz4lpNXaI3Xj1FqJBGzzgVc\&s=qOrBEztXzKSspdg-HIxuw0tq0vBnjIuULrAxoe7ab5o\&e=

Mladenović, I., Milovančević, M., Sokolov-Mladenović, S.: Analyzing of innovations influence on economic growth by fuzzy system (2016a). Published 17 March 2016. https://urldefense.proofpoint.com/ v2/url?u=https-3A_doi.org_10.1007_s11135-2D016-2D0331-2D4\&d=DwIFaQ\&c=vh6FgFndue jNhPPD0f1_yRaSfZy8CWbWnIf4XJhSqx8\&r=mImVkBpa54MY3KIaquiNBAiNRIkbDDT3A20

The original article can be found online at https://doi.org/10.1007/s11135-016-0337-y.

Igor Mladenović

igor.mladenovic@eknfak.ni.ac.rs

1 Department of Biotechnology, University of Rijeka, Rijeka, Croatia

2 University of Niš, Faculty of Economics, Trg kralja Aleksandra 11, Niš 18000, Serbia

3 Young Researchers and Elites Club, Hamedan Branch, Islamic Azad University, Hamedan, Iran 
7pVhZWFk\&m=61Q4FOkv2cm-bbmO9NhTz4lpNXaI3XjlFq-JBGzzgVc\&s=Bv0GRr8RAYDNaS1 qW6L3bI6ru7VeDm8onxyxy_mBYKE\&e=

Mladenović, I., Marković, D., Milovančević, M., Nikolić, M.: Extreme learning approach with wavelet transform function for forecasting wind turbine wake effect to improve wind farm efficiency (2016b). Published 29 March 2016. https://urldefense.proofpoint.com/v2/url?u=https-3A_doi.org_10.1016_j. advengsoft.2016.02.011\&d=DwIFaQ\&c=vh6FgFnduejNhPPD0f1_yRaSfZy8CWbWnIf4XJhS qx8\&r=mImVkBpa54MY3KIaquiNBAiNRIkbDDT3A207pVhZWFk\&m=61Q4FOkv2cm-bbmO9 NhTz4lpNXaI3XjlFq-JBGzzgVc\&s=VlNQBA4UruKf1W9a8NAr2mbnlzMt-e0Ri4qvrDAv8Qk\&e=

Publisher's Note Springer Nature remains neutral with regard to jurisdictional claims in published maps and institutional affiliations. 